\title{
Evaluation des propriétés antimicrobiennes de quatre plantes de la flore togolaise utilisées en médecine traditionnelle dans le traitement des diarrhées infantiles
}

\author{
Yao Patrick HOEKOU ${ }^{1,3^{*}}$, Komlan BATAWILA ${ }^{2}$, Koffi Apeti GBOGBO ${ }^{2}$, \\ Damintoti Simplice KAROU ${ }^{3}$, Yaovi AMEYAPOH ${ }^{3}$ et Comlan SOUZA ${ }^{3}$ \\ ${ }^{1}$ Laboratoire de Microbiologie de l'Ecole Supérieure des Techniques Biologiques et Alimentaires (ESTBA), \\ Université de Lomé, Togo. \\ ${ }^{2}$ Laboratoire de Botanique et Ecologie Végétale, Faculté de Sciences, Université de Lomé, Togo. \\ ${ }^{3}$ Centre de Formation et de Recherche sur les Plantes Médicinales (CERFOPLAM), Université de Lomé, Togo. \\ *Auteur correspondant, E-mail: patrick21h@yahoo.fr ; BP 1515 Lomé; Togo.
}

\section{RESUME}

Ce travail a été entrepris afin de justifier l'utilisation traditionnelle de quatre plantes de la flore togolaise dans le traitement des diarrhées infantiles. Les potentiels antibactériens et antifongiques des extraits hydroéthanoliques des feuilles de Byrsocarpus coccineus, Leptadenia hastata, Phyllanthus muellerianus, Pupalia lappacea, et d'écorces de racine de Phyllanthus muellerianus, ont été évalués in vitro par la méthode de dilution en milieu liquide associée à l'étalement sur milieux gélosés. Les germes : Escherichia coli, Shigella flexneri, Salmonella typhi, Salmonella enteritidis et Candida albicans, souvent incriminés dans les maladies diarrhéiques infantiles ont été testés. Les extraits hydroéthanoliques de ces plantes ont inhibé à divers degrés la croissance in vitro des germes testés, à l'exception de ceux des feuilles de P. lappacea et de B. coccineus qui ont été inactifs respectivement sur $C$. albicans et $S$. flexneri. Les concentrations minimales inhibitrices des extraits ont varié de 0,5 à $4 \mathrm{mg} / \mathrm{ml}$. Le test phytochimique a montré que tous ces extraits contiennent des alcaloïdes, des flavonoïdes, des tanins et des saponosides, sauf les extraits des feuilles de $P$. lappacea et de $L$. hastata qui ne possèdent respectivement pas d'alcaloïdes et de tanins.

(C) 2012 International Formulae Group. All rights reserved.

Mots clés: Infections diarrhéiques, extraits végétaux, antimicrobiens, phytochimie.

\section{INTRODUCTION}

Depuis 1978, date à laquelle l'Organisation Mondiale de la Santé (OMS) et le Fonds des Nations Unies pour l'Enfance (UNICEF) ont adopté la thérapie par réhydratation orale, comprenant l'administration de solutions de sels de réhydratation orale comme outil principal de la lutte contre la déshydratation liée aux diarrhées, la mortalité des enfants âgés de moins de 5 ans atteints de diarrhée est passée de 4,5 millions à 0,8 million par an (Bryce et al., 2005; Liu et al., 2012). Malgré ces résultats significatifs, la diarrhée demeure toujours l'une des principales causes de décès des enfants dans les pays en développement (Fontaine, 2008).

Deuxième cause de mortalité infantile dans le monde après les pneumopathies, et en Afrique après le paludisme, la diarrhée 
demeure un problème majeur de santé publique. Au Togo, elle représente $15 \%$ de la mortalité infantile, troisième cause, derrière le paludisme et les pneumopathies qui représentent respectivement $19 \%$ et $18 \%$ (OMS, 2011).

Face à ce problème qu'est la diarrhée, près de $80 \%$ de la population du Togo, à l'instar de l'ensemble des pays en développement, utilisent encore la médecine traditionnelle pour se soigner (WHO, 2009). Il s'avère donc nécessaire d'étudier les potentialités antimicrobiennes des plantes d'usage traditionnel afin de valider leur utilisation par les populations ou par les tradipraticiens, mais aussi avec l'espoir de trouver en elles des médicaments efficaces, peu onéreux et accessibles à tous.

Chez les Adja et Ouatchi du Sud Togo, diverses plantes médicinales sont fréquemment utilisées dans le traitement des infections diarrhéiques, et celles-ci incluent: Byrsocarpus coccineus Schum. et Thonn (Connaraceae), Leptadenia hastata (Pers.) Decne (Asclepiadaceae), Phyllanthus muellerianus (Kuntze) Exell (Euphorbiaceae), et Pupalia lappacea (Linn.) Juss (Amaranthaceae). Ces quatre plantes sont diversement utilisées en médecine traditionnelle au Togo, en Afrique et partout dans le monde. $P$. muellerianus est couramment utilisée pour traiter le paludisme et les troubles intestinaux. L'infusion de jeunes pousses sert à traiter la dysenterie sévère (Adjanohoun et al., 1986). Les racines sont infusées dans de l'eau et le liquide se boit pour traiter la diarrhée. Les racines bouillies à l'eau s'emploient aussi en lavement pour traiter les maux d'estomac. La macération de feuilles et de racines s'utilise comme lotion du corps pour traiter les fièvres éruptives infantiles (Ben-Baala, 2008). Les décoctions et les macérations de racines et de feuilles de L. hastata sont utilisées contre les douleurs abdominales, les écoulements urétraux, les maux d'estomac, la diarrhée, la gonorrhée, l'agalactie, les anuries et l'impuissance sexuelle (Berhaut, 1971; Jansen, 2004). Les feuilles de B. coccineus sont utilisées dans le traitement de l'hypertension artérielle, de la gonococcie, de la dysenterie, de l'impuissance sexuelle, de la flatulence, de la jaunisse, des maladies vénériennes, des douleurs buccales et de la peau (Burkill, 1985). P. lappacea est utilisée en décoction avec Piliostigma thonningii, comme boisson antidysentérique. P. lappacea est aussi utilisée pour traiter les œdèmes de jambe, la toux et la fièvre. Les cendres de la plante brûlée sont mélangées avec de l'eau et données en boisson contre la flatulence (Berhaut, 1971).

Des études antérieures ont montré que ces plantes possèdent de multiples propriétés pharmacologiques (Akindele et Adeyemi, 2010 ; Ndjonka et al., 2010; Assob et al., 2011 ; Brusotti et al., 2011) mais peu d'entre elles ont porté sur leurs propriétés antimicrobiennes vis-à-vis des germes responsables de maladies diarrhéiques. La revue de littérature ne révèle à notre connaissance aucune étude sur l'activité antimicrobienne de P. lappacea.

La présente étude, qui s'inscrit dans le cadre de la valorisation des plantes médicinales de la flore togolaise a pour but d'évaluer les propriétés inhibitrices des extraits de ces quatre plantes vis-à-vis des microorganismes responsables des maladies diarrhéiques chez les enfants, et d'identifier par une analyse sommaire les grands groupes chimiques présents dans les extraits de ces plantes.

\section{MATERIEL ET METHODES Matériel végétal}

Les organes de plantes ont été récoltés en octobre 2010 dans les villages de Hognonkondji, Agodomé et Zouvi (préfecture de Yoto au Togo) après les enquêtes ethnobotaniques puis étiquetés et ramenés au laboratoire de Botanique et Ecologie Végétale de la Faculté des Sciences de l'Université de Lomé, où ils ont été identifiés. Il s'agi des feuilles de B. coccineus, de P. lappacea, et de $L$. hastata, et des feuilles et écorces de racine de P. muellerianus.

\section{Souches microbiennes}

Les souches bactériennes utilisées pour les tests ont été isolées et identifiées au 
laboratoire de Bactériologie du Centre Hospitalier Universitaire Sylvanus Olympio à Lomé. La souche fongique provient du laboratoire de bactériologie de l'Institut National d'Hygiène de Lomé. Les souches bactériennes sont : E. coli, S. typhi, S. enteritidis, $S$. flexneri, et la souche fongique est $C$. albicans. Ces souches ont été isolées des selles diarrhéiques chez des enfants.

\section{Extraction}

Les différents organes de plantes (feuilles, écorces de racines) sont finement coupés et séchés au laboratoire à la température ambiante. Ils sont broyés puis réduits en poudre grâce à un moulin électrique. Cent (100) g de poudre de feuilles ou d'écorce de racine sont macérés dans l'éthanol $70 \%(\mathrm{~V} / \mathrm{V})$ pendant 48 à 72 heures sous agitation continue. Le produit obtenu est filtré sur papier Whatman $\mathrm{n}^{\circ} 1$, puis évaporé à sec. Les extraits récupérés sont conservés à 4 ${ }^{\circ} \mathrm{C}$ au réfrigérateur avant les tests.

\section{Tests antimicrobiens}

Les tests antimicrobiens ont été effectués selon la technique décrite par de Souza et al. (1993) ; et reprise par Agassounon et al. (2001).

\section{Préparation de la suspension microbienne}

Une colonie de 24 heures est prélevée à l'aide d'une anse stérile et inoculée dans 10 ml de bouillon Mueller Hinton pour les bactéries ou bouillon Sabouraud Chloramphénicol pour la levure. Un prélèvement d'une aliquote de $1 \mathrm{ml}$ de cette suspension bien homogénéisée est effectué et dilué à $10^{-3}$ dans du bouillon Mueller Hinton pour les bactéries ou bouillon Sabouraud Chloramphénicol pour la levure pour avoir la suspension microbienne qui a servi à la réalisation des tests. Dix (10) $\mu 1$ de cette suspension ont été ensemencés par étalement sur gélose Mueller Hinton pour les souches bactériennes ou gélose Sabouraud Chloramphénicol pour la souche fongique. Les boîtes sont incubées à $37{ }^{\circ} \mathrm{C}$ pendant 24 heures pour les bactéries et pendant 48 heures pour la souche fongique puis les colonies sont dénombrées en vue de déterminer la charge microbienne qui est mise en contact des extraits.

\section{Préparation des solutions d'extraits}

Les extraits totaux hydroéthanoliques obtenus, ont servi à préparer des solutions de concentration $40 \mathrm{mg} / \mathrm{ml}$, qui ont été stérilisées par filtration à l'aide de seringues-filtre sur membrane millipore $0,22 \mu \mathrm{m}$. La stérilité des solutions d'extraits a été vérifiée en ensemençant des aliquotes de chaque solution sur les milieux Mueller Hinton et Sabouraud+Chloramphénicol qu'on a incubé à $37^{\circ} \mathrm{C}$ pendant 24 à 48 heures.

Test présomptif

La méthode de dilution en milieu liquide couplée à l'étalement sur milieux gélosés a été utilisée comme décrite par de Souza et al. (1993), et reprise par Agassounon Djikpo Tchibozo et al. (2001). Le test présomptif consiste à utiliser une seule concentration des extraits pour identifier les extraits actifs. L'essai a été constitué en introduisant dans un tube à hémolyse stérile, $0,5 \mathrm{ml}$ de l'extrait à tester et $10 \mu \mathrm{l}$ de la suspension microbienne. Dans le tube témoin, l'extrait a été substitué par $0,5 \mathrm{ml}$ de bouillon nutritif stérile. Un témoin positif a été réalisé dans les mêmes conditions en utilisant la gentamicine $(0,015 \mathrm{mg} / \mathrm{ml})$ pour les bactéries et la nystatine $(0,10 \mathrm{mg} / \mathrm{ml})$ pour $C$. albicans. Les tubes ainsi constitués sont incubés à $37^{\circ} \mathrm{C}$ pendant 24 à 48 heures puis les essais et les témoins sont étalés sur milieux gélosés appropriés à raison de deux boîtes par tube. Les milieux gélosés sont ensuite incubés à 37 ${ }^{\circ} \mathrm{C}$ pendant 24 à 48 heures. Les colonies sont alors comptées sur chaque boîte et les pourcentages de survivance et d'inhibition sont calculés par rapport au témoin négatif selon la formule : $\mathbf{P I}=\mathbf{1 0 0}(\mathbf{1}-\mathbf{X} / \mathbf{Y})$

PI : Pourcentage d'inhibition. ; $\mathbf{X}$ : nombre de colonies du germe dénombré sur la boîte test. Y : nombre de colonies du germe dénombré sur la boîte témoin.

\section{Test pour l'établissement de la courbe de sensibilité}

Ce test dépend des résultats du test présomptif et a été effectué lorsque le test présomptif a révélé que l'extrait inhibe totalement le germe étudié. A partir d'une 
solution mère, une série de dilutions successives par progression géométrique de raison 2 a été effectuée de manière à obtenir une gamme de concentrations finales dans les tubes comprise entre 32 et $0,0625 \mathrm{mg} / \mathrm{ml}$. La procédure reste la même que pour le test présomptif. La concentration minimale inhibitrice (CMI) a été déterminée à l'œil nu, et correspond donc à la plus faible concentration de l'extrait pour laquelle aucune croissance n'est visible. Le contenu de tous les tubes où il n'y a pas eu de croissance a été ensemencé sur milieu Mueller Hinton pour les bactéries et Sabouraud Chloramphénicol pour la levure, puis incubé à $37{ }^{\circ} \mathrm{C}$ pendant 24 heures. L'analyse de ces résultats nous a permis de déterminer la concentration minimale bactéricide (CMB) ou fongicide (CMF) qui est égale à la concentration d'extrait qui tue $99,99 \%$ du germe en culture.

\section{Rapport CMB/CMI}

Les CMI et les CMB ou CMF déterminées sont caractéristiques d'un extrait pour une souche donnée. Ainsi donc, l'action d'un extrait sera considérée comme bactéricide si le rapport $\mathrm{CMB} / \mathrm{CMI}$ est égal à 1 . L'action est dite bactériostatique si le rapport $\mathrm{CMB} / \mathrm{CMI}$ est supérieur à 1 (Karou et al., 2005).

\section{Etudes phytochimiques}

La recherche des grands groupes chimiques dans les extraits végétaux a été faite par une analyse phytochimique qualitative sommaire à partir des tests de coloration (Harbone, 1976).

\section{Mise en évidence des alcaloüdes}

Méthode 1: Les alcaloïdes ont été identifiés par le réactif de Mayer (10 g de KI et $2,70 \mathrm{~g}$ de $\mathrm{HgCl}_{2}$ dissous dans $20 \mathrm{ml}$ d'eau). L'ajout de quelques gouttes de ce réactif à 2 $\mathrm{ml}$ de la solution d'extrait entraîne la formation d'un précipité blanc ou blanc-jaune en présence des alcaloïdes.

Méthode 2: Le réactif de Bouchardat (solution de $2 \mathrm{~g}$ d'iode bisublimé et $2 \mathrm{~g}$ de KI dans $100 \mathrm{ml}$ d'eau distillée) est également utilisé pour mettre en évidence la présence des alcaloïdes dans les extraits. Il se forme un précipité brun-noir, brun-terre ou jaune brun avec les alcaloïdes.

\section{Mise en évidence des flavonoüdes}

Méthode 1: Dans un tube, on a ajouté à quelques $\mathrm{ml}$ de la solution d'extrait, quelques gouttes d'une solution de soude au 1/10. La coloration jaune-orange signale la présence des flavonoïdes.

Méthode 2: Deux à 3 gouttes d'une solution diluée de perchlorure de fer $\left(\mathrm{FeCl}_{3}\right)$ sont ajoutées à quelques $\mathrm{ml}$ de solution d'extrait dans un tube à essai. L'observation d'une coloration verdâtre indique la présence des flavonoïdes.

\section{Mise en évidence des saponosides}

Après agitation pendant une minute d'un tube à essai contenant quelques $\mathrm{ml}$ d'extrait aqueux, il se forme une mousse persistante d'environ $1 \mathrm{~cm}$ de hauteur en présence des saponosides.

\section{Mise en évidence des tanins}

Technique: Réaction à l'acétate de plomb 10\%: Un ml de la solution aqueuse d'acétate de plomb à $10 \%$ est ajouté à $3 \mathrm{ml}$ d'extrait. La formation d'un précipité bleu, bleu-noir, blanchâtre ou brunâtre indique la présence des tanins.

\section{RESULTATS}

Activités inhibitrices des différents extraits hydroéthanoliques à la concentration de 40 mg/ml

Le Tableau 1 nous présente les pourcentages d'inhibition des différents extraits hydroéthanoliques testés. A la concentration de $40 \mathrm{mg} / \mathrm{ml}$, l'extrait hydroéthanolique des feuilles de $P$. muellerianus a inhibé totalement la croissance in vitro de $E$. coli et $C$. albicans; et a eu une action forte contre S. typhi (92,3\%), Shigella flexneri $(85 \%)$, et une action moyenne contre S. enteritidis $(56,5 \%)$. L'extrait d'écorce de racine de $P$. muellerianus a inhibé totalement la croissance de $S$. flexneri et $C$. albicans. Il a eu une action moyenne contre $E$. coli $(59,2 \%)$, $S$. typhi $(60 \%)$, et $S$. enteritidis $(64,5 \%)$. L'extrait hydroéthanolique des feuilles de $P$. lappacea à $40 \mathrm{mg} / \mathrm{ml}$ a inhibé totalement la croissance de E. coli, S. typhi et S. enteritidis, mais est inactif sur $C$. albicans. Il a une faible activité inhibitrice $(35 \%)$ contre Shigella 
flexneri. L'extrait hydroéthanolique des feuilles de B. coccineus a inhibé totalement la croissance de E. coli, S. typhi, S. enteritidis et C. albicans; alors que son action est faible contre S. flexneri (25\%). L'extrait hydroéthanolique des feuilles de L. hastata a inhibé totalement la croissance de E. coli seule sur les 5 souches testées, mais a inhibé fortement la croissance de S. typhi $(91 \%)$ et $S$. enteritidis $(93,5 \%)$; et moyennement celle de Shigella flexneri (53\%) et C. albicans $(62,5 \%)$. Les drogues de référence, la gentamicine à $15 \mu \mathrm{g} / \mathrm{ml}$ pour les souches bactériennes et la nystatine à $100 \mu \mathrm{g} / \mathrm{ml}$ pour la souche de Candida albicans, ont montré une inhibition totale.

Concentrations minimales inhibitrices (CMI) et concentrations minimales bactéricides (CMB) et fongicides (CMF) des différents extraits, et leur interprétation Les concentrations minimales inhibitrices (CMI) et les concentrations minimales bactéricides (CMB) et fongicides (CMF) des extraits ayant montré une inhibition totale à $40 \mathrm{mg} / \mathrm{ml}$ sont présentées dans le Tableau 2.

Les concentrations minimales inhibitrices des extraits hydroéthanoliques testés varient de $0,5 \mathrm{mg} / \mathrm{ml}$ à $4 \mathrm{mg} / \mathrm{ml}(0,5$ à 2 $\mathrm{mg} / \mathrm{ml}$ pour les souches bactériennes, et 2 à 4 $\mathrm{mg} / \mathrm{ml}$ pour la souche de $C$. albicans); et les concentrations minimales bactéricides de 1 à 2,5 $\mathrm{mg} / \mathrm{ml}$ alors que les concentrations minimales fongicides varient de 2,5 à 4 $\mathrm{mg} / \mathrm{ml}$. L'extrait des feuilles de B. coccineus est le plus actif (inhibition totale contre 4 germes sur 5) avec des CMI allant de 1 à 2 $\mathrm{mg} / \mathrm{ml}$ et $E$. coli est le germe le plus sensible à tous nos extraits de plantes (CMI variant de $0,5$ à $1 \mathrm{mg} / \mathrm{ml})$. L'interprétation des résultats nous a montré que les extraits testés ont eu un effet bactériostatique sur les souches bactériennes testées. Les extraits de feuilles de $B$. coccineus et de $P$. muellerianus ont eu un effet fongicide sur la souche de $C$. albicans alors que les extraits d'écorce de racine de $P$. muellerianus ont eu un effet fongistatique.

\section{Etudes phytochimiques}

L'étude phytochimique nous a permis d'identifier quelques grands groupes chimiques dans les extraits d'organes de plantes testés. Les résultats sont consignés dans le Tableau 3.

L'analyse de ce tableau montre que tous les extraits contiennent des alcaloïdes, des flavonoïdes, des tanins et des saponosides, sauf les extraits hydroéthanoliques des feuilles de $P$. lappacea et de L. hastata qui ne contiennent respectivement pas d'alcaloïdes et de tanins.

Tableau 1: Activités inhibitrices des différents extraits hydroéthanoliques testés en pourcentage.

\begin{tabular}{lccccc}
\hline Microorganismes Extraits & E. coli & S. flexneri & S. typhi & S. enteritidis & C. albicans \\
\hline PmF & 100 & 85 & 92,3 & 56,5 & 100 \\
PmER & 59,2 & 100 & 60 & 64,5 & 100 \\
PIF & 100 & 35 & 100 & 100 & 00 \\
$\mathrm{BcF}$ & 100 & 25 & 100 & 100 & 100 \\
$\mathrm{LhF}$ & 100 & 53 & 91 & 93,5 & 62,5 \\
Gentamicine $(15 \mu \mathrm{g} / \mathrm{ml})$ & 100 & 100 & 100 & 100 & $\mathrm{NT}$ \\
Nystatine $(100 \mu \mathrm{g} / \mathrm{ml})$ & $\mathrm{NT}$ & $\mathrm{NT}$ & $\mathrm{NT}$ & $\mathrm{NT}$ & 100 \\
\hline
\end{tabular}

PmF = Feuilles de Phyllanthus muellerianus $;$ PmER = Ecorce de racine de Phyllanthus muellerianus $; \quad$ PIF = Feuilles de Pupalia lappacea $; \mathrm{BcF}=$ Feuilles de Byrsocarpus coccineus $; \mathrm{LhF}=$ Feuilles de Leptadenia hastata. $\mathrm{NT}=$ non testé. 
Tableau 2: Concentrations minimales inhibitrices (CMI) et concentrations minimales bactéricides (CMB) ou fongicides (CMF) des extraits en $\mathrm{mg} / \mathrm{ml}$.

\begin{tabular}{|c|c|c|c|c|c|}
\hline Extraits & Souches & CMI (mg/ml) & $\mathrm{CMB}(\mathrm{mg} / \mathrm{ml})$ & CMB/CMI & Interprétation \\
\hline \multirow[b]{2}{*}{$\mathrm{PmF}$} & E. coli & 0,5 & 1 & 2 & bactériostatique \\
\hline & C. albicans & 4 & 4 & 1 & fongicide \\
\hline \multirow[b]{2}{*}{ PmER } & S. flexneri & 2 & 2,5 & 1,25 & bactériostatique \\
\hline & C. albicans & 2 & 2,5 & 1,25 & fongistatique \\
\hline \multirow{3}{*}{$\mathrm{PlF}$} & E. coli & 1 & 2 & 2 & bactériostatique \\
\hline & S. typhi & 1 & 1,5 & 1,25 & bactériostatique \\
\hline & S. enteritidis & 2 & 2,5 & 1,25 & bactériostatique \\
\hline \multirow{4}{*}{$\mathrm{BcF}$} & E. coli & 1 & 1,5 & 1,25 & bactériostatique \\
\hline & S. typhi & 2 & 2,5 & 1,25 & bactériostatique \\
\hline & S. enteritidis & 1 & 1,5 & 1,5 & bactériostatique \\
\hline & C. albicans & 4 & 4 & 1 & fongicide \\
\hline LhF & E. coli & 1 & 1,5 & 1,5 & bactériostatique \\
\hline
\end{tabular}

Tableau 3: Composition chimique des différents extraits.

\begin{tabular}{lcccc}
\hline & Tanins & Flavonoïdes & Alcaloïdes & Saponosides \\
\hline P. muellerianus $(\mathrm{F})$ & ++ & + & + & ++ \\
P. muellerianus $(\mathrm{ER})$ & ++ & ++ & + & +++ \\
Pupalia lappacea $(\mathrm{F})$ & + & ++ & - & +++ \\
B. coccineus $(\mathrm{F})$ & ++ & + & ++ & ++ \\
Leptadenia hastata $(\mathrm{F})$ & - & ++ & ++ & + \\
\hline \multicolumn{2}{l}{ - = absence, $+=$ présence,,$++=$ peu important,,$+++=$ important, $(\mathrm{F})=$ feuilles, $(\mathrm{ER})=$ écorces de racines }
\end{tabular}

\section{DISCUSSION}

Dans ce travail, nous avons évalué les activités contre $C$. albicans, et 4 souches bactériennes souvent incriminées dans les maladies diarrhéiques infantiles. La découverte de plantes actives contre ces agents de maladies peut être profitable pour la santé publique dans les pays pauvres tels que le Togo. Toutes les espèces végétales testées dans ce travail sont prometteuses.

Les extraits de feuilles et d'écorce de racine de $P$. muellerianus ont diversement inhibé in vitro la croissance de tous les microorganismes testés. L'extrait de feuilles de $P$. muellerianus a eu une action bactériostatique sur la croissance de E. coli. $S$. flexneri est plus sensible à l'extrait d'écorce de racine qu'à l'extrait de feuilles de $P$. 
muellerianus. L'extrait de feuilles a eu une activité fongicide contre C. albicans alors que l'extrait d'écorce de racine a montré une activité fongistatique. Ces résultats peuvent s'expliquer par le fait que ces deux extraits n'ont pas la même composition phytochimique. La littérature nous montre que les extraits de feuilles de $P$. muellerianus contiennent des tanins, des flavonoïdes, des saponines, des alcaloïdes et des anthraquinones (Ndjonka et al., 2010), qui posssèdent d'énormes potentialités antimicrobiennes. Nos résultats sont proches de ceux de Onocha et al. (2003) qui ont montré que l'extrait au chloroforme des feuilles de P. muellerianus a une forte activité antifongique contre $C$. albicans ainsi qu'une activité antibactérienne contre Escherichia coli. Katsayal et Lamai, (2009), et Assob et al. (2011) ont déjà montré une forte activité antibactérienne de l'extrait d'écorce de tige de P. muellerianus contre E. coli, S. typhi, S. flexneri, et une activité antifongique contre C. albicans. Par contre, Brusotti et al. (2011) ont montré que les extraits aqueux et méthanolique d'écorce de tige de $P$. muellerianus sont inactifs sur Escherichia coli ATCC 10536, et C. albicans ATCC 10231. Les propriétés antimicrobiennes de l'écorce de racine de $P$. muellerianus sont rapportées ici pour la première fois.

L'extrait hydroéthanolique des feuilles de $P$. lappacea a eu une activité bactériostatique sur E. coli, S. typhi et $S$. enteritidis mais il est inactif sur C. albicans. L'analyse phytochimique a montré que cet extrait contient des saponosides, des tanins et des flavonoïdes. Ces substances sont reconnues pour leurs propriétés antimicrobiennes et justifient donc les résultats obtenus. Ndjonka et al. (2010) ont déjà révélé la présence de flavonoïdes, saponines, carbohydrates et tanins dans les extraits de feuilles de P. lappacea. A notre connaissance, la littérature relative aux activités antimicrobiennes de P. lappacea était manquante jusqu'à nos travaux.

L'extrait hydroéthanolique des feuilles de B. coccineus a montré une activité bactériostatique sur E. coli, $S$. typhi et $S$. enteritidis ; et une activité fongicide sur $C$. albicans. La présence des flavonoïdes, saponosides, alcaloïdes et tanins dans l'extrait de feuilles de B. coccineus serait à l'origine de ces activités antimicrobiennes. Ces résultats viennent appuyer les travaux de Ahmadu et al. (2006) qui ont montré que les extraits à l'acétate d'éthyle et au n-butanol des feuilles de $B$. coccineus ont inhibé la croissance de $E$. coli (CMI : $1,75 \mathrm{mg} / \mathrm{ml})$, S. typhi (CMI : 1,75 $\mathrm{mg} / \mathrm{ml}$ ), et C. albicans (CMI : $0,88 \mathrm{mg} / \mathrm{ml}$ ). Akindele et Adeyemi (2006), ont montré l'activité antidiarrhéique de $B$. coccineus par des tests physiologiques. L'activité antibactérienne contre $S$. enteritidis est rapportée ici pour la première fois.

L'extrait hydroéthanolique des feuilles de L. hastata a montré une activité bactériostatique contre $E$. coli et a diversement inhibé la croissance des autres microorganismes testés. Les tests phytochimiques montrent que cet extrait contient des alcaloïdes, flavonoïdes et saponosides qui sont des substances antimicrobiennes. Nos résultats sont similaires à ceux déjà obtenus contre $E$. coli par Aliero et Wara (2009), qui ont trouvé que L. hastata a inhibé la croissance de $E$. coli avec une concentration de $30 \mathrm{mg} / \mathrm{ml}$. Quant au potentiel antibactérien de l'extrait des feuilles de cette plante contre $S$. typhi, $S$. enteritidis, et S. flexneri, à notre connaissance, c'est la première fois qu'il est observé mais il a été déjà recherché contre Salmonella paratyphi. Soro et al. (2010) ont par contre montré que l'extrait méthanolique des tiges feuillées de $L$. hastata, à la concentration de $100 \mu \mathrm{g} / \mathrm{ml}$, est inactif sur $C$. albicans.

\section{Conclusion}

La présente étude nous a montré que les extraits hydroéthanoliques des feuilles de L. hastata (Pers.) Decne, P. muellerianus (Kuntze) Exell, P. lappacea (Linn.) Juss et $B$. coccineus Schum. et Thonn, inhibent diversement in vitro la croissance des microorganismes testés et contiennent des composés chimiques responsables d'activités 
antimicrobiennes. Ces résultats confirment les activités antibactérienne et antifongique de ces quatre plantes, et justifient leurs utilisations dans la thérapeutique traditionnelle antidiarrhéique au Togo. Ces plantes pourraient donc servir de matières premières à la fabrication de médicaments pour le traitement des infections microbiennes.

\section{REFERENCES}

Adjanohoun EJ, Ahyi MRA, Ake Assi L, Akpagana K, Chibon P, El-Hadj Watar A, Eyme J, Garba M, Gassita JN, Gbeassor M, Goudote E, Guinko S, Hodouto KK, Houngnon P, Keita A, Keoula Y, Kluga-Ocloo WP, Lo I, Siamevi KM, Taffame KK. 1986. Contribution aux études ethnobotaniques et floristiques au Togo. Médecine traditionnelle et pharmacopée. Agence de Coopération Culturelle et Technique, Paris.

Agassounon Djikpo TTM, de Souza C, Anani KT, Koumaglo K, Toukourou F, Gbeassor M. 2001. Evaluation des activités cytotoxique, antivirale, antibactérienne et antifongique de six plantes médicinales. Pharm. Méd. Afr., 11: 93-105.

Ahmadu AA, Akpulu IN, Hassan HS, Sule MI, Pateh UU. 2006. Preliminary phytochemical and antimicrobial screening of the leaves of Byrsocarpus coccineus Schum and Thonn. (Connaraceae). Journal of Pharmaceutical Bioresources, 3(2): 107110.

Akindele AJ, Adeyemi OO. 2006. Evaluation of the antidiarrhoeal activity of Byrsocarpus coccineus. Journal of Ethnopharmacology, 108: 20-25.

Akindele AJ, Adeyemi OO. 2010. Anxiolytic and sedative effects of Byrsocarpus coccineus Schum. and Thonn. (Connaraceae) extrac. International Journal of Applied Research in Natural Products, 3(1): 28-36.

Aliero AA, Wara SH. 2009. Validating the medicinal potential of Leptadenia hastata. African Journal of Pharmacy and Pharmacology, 3(6): 335-338.

Assob JCN, Kamga HLF, Nsagha DS, Njunda AL, Nde PF, Asongalem EA, Njouendou AJ, Sandjon B, Penlap VB. 2011. Antimicrobial and toxicological activities of five medicinal plant species from Cameroon Traditional Medicine. BMC Complementary and Alternative Medicine, 11: 70.

Ben-Bala KD. 2008. Phyllanthus muellerianus (Kuntze) Exell. Fiche de Protabase, Schmelzer, G.H, Gurib-Fakim A (eds). PROTA (Plant Resources of Tropical Africa / Ressources végétales de l'Afrique tropicale): Wageningen, Pays Bas.

Berhaut J. 1971. Flore Illustrée du Sénégal. Dicotylédones (Tome I). 185-186 ; 545547.

Brusotti G, Cesari I, Frassa G, Grisoli P, Daccaro C, Caccialanza G. 2011. Antimicrobial properties of stem bark extracts from Phyllanthus muellerianus (Kuntze) Excell. Journal of Ethnopharmacology, 135(3): 797-800.

Bryce J, Boschi-Pinto C, Shibuya K. 2005. WHO estimates of the causes of death in children. Lancet, 365: 1147-1152.

Burkill HM. 1997. The Useful Plants of West Tropical Africa (2 ${ }^{\text {nd }}$ edn, Vol. 2). Royal Botanic Gardens: Kew, United Kingdom.

de Souza C, Ameganvi KK, Koumaglo K, Gbeassor M. 1993. Etude de l'activité antimicrobienne des extraits aqueux totaux de dix plantes médecinales. Revue de Médecines et Pharmacopées Africaines, 2(7): 107-115.

Fontaine O. 2008. Nouvelles recommandations de l'OMS pour la prise en charge clinique de la diarrhée. La nouvelle formule de sels de réhydratation par voie orale et l'administration de Zinc. Pediatrica, 19(5): 25-27.

Harbone JB. 1976. Phytochemical Methods. A Guide to Modern Techniques of Plant Analyses. Chapman and hall: London.

Jansen PCM. 2004. Leptadenia hastata (Pers.) Decne, Grubben GJH, Denton 
OA (eds). PROTA 2: Vegetables/Légumes. [CD-Rom]. PROTA: Wageningen, Pays Bas.

Karou D, Dicko MH, Simpore J, Traore AS. 2005. Antioxidant and antibacterial activities of polyphenols from ethnomedicinal plants of Burkina Faso. African Journal of Biotechnology, 4(8): 823-828.

Katsayal AU, Lamai RS. 2009. Preliminary phytochemical an antibacterial screening of the ethanolic stem bark extract of Phyllanthus muellerianus. Nig. Journ. Pharm. Sci., 8(2): 121-125.

Liu L, Johnson HL, Cousens S, Perin J, Scott S, Lawn JE, Rudan I, Campbell H, Cibulskis R, Li M, Mathers C, Black RE. 2012. Global, regional, and national causes of child mortality: an updated systematic analysis for 2010 with time trends since 2000. Lancet, 379: 21512161

Ndjonka D, Agyare C, Lüersen K, Hensel A,
Liebau E. 2010. In vitro anti-leishmanial activity of traditional medicinal plants from Cameroon and Ghana. Int. J. Pharmacol., 6(6): 863-871.

OMS. 2011. Statistiques sanitaires mondiales. OMS, 171p.

Onocha PA, Opegbemi AO, Kadri AO, Ajayi KM, Okorie DA. 2003. Antimicrobial evaluation of Nigerian Euphorbiaceae plants : Phyllanthus amarus and Phyllanthus muellerianus leaf extracts. Nigerian Journal of Natural Products and Medicine, 7: 9-12.

Soro D, Koné MW, Kamanzi K. 2010. Evaluation des activités antimicrobiennes et anti-radicaux libres de quelques taxons bioactifs de Côte D'ivoire. European Journal of Scientific Research, 40(2): 307-317.

WHO. 2009. Traditional medicine; Available online from http://www.who.int/topics/ tradidional_ medicine/en/ Accessed on October 2012. 